\title{
Step counts and self-reported physical activity among upper elementary school students vary with aerobic fitness
}

\author{
Annie McGee ${ }^{1}$, Skip M. Williams², Margaret M. Coleman², Amy Hurd², Kelly R. Laurson² \\ ${ }^{1}$ Hudson Elementary School, Hudson, IL, ${ }^{2}$ Illinois State University, Normal, IL
}

\section{Summary}

Study aim: The purpose of this study was to examine if step-counts during PE and self-reported PA of elementary grade students varied based on the aerobic capacity.

Material and methods: Ninety elementary physical education students, enrolled in the $4^{\text {th }}$ and $5^{\text {th }}$ grade, from one elementary school in the Midwestern USA participated. Each participant completed the Physical Activity Questionnaire for Children (PAQ-C), wore a pedometer in PE to measure steps taken, and completed the PACER aerobic fitness test. A multiple regression analysis was conducted to determine the relationship between steps taken by students and PAQ-C score in predicting aerobic capacity as measured through the PACER test.

Results: Average steps significantly predicted PACER laps $(\beta=0.48, \mathrm{p}<0.01)$, as did the PAQ-C $(\beta=0.28, \mathrm{p}<0.001)$. For males, average steps significantly predicted PACER laps $(\beta=0.48, p<0.01)$, while the PAQ-C and the PACER beta coefficients were not statistically significant $(\beta=0.14, p=0.30)$. Results for females indicated average steps significantly predicted PACER laps $(\beta=0.38, \mathrm{p}<0.01)$, as did the PAQ-C $(\beta=0.46, \mathrm{p}<0.001)$.

Conclusion: Results from this study indicate that student steps taken during PE and self-report PA has an association with students' completed PACER laps.

Keywords: Physical Activity - Physical Education - Aerobic Capacity - PAQ-C - Pedometers

\section{Introduction}

With the current sedentary lifestyle trends, it is not surprising that in 2009 only $18.4 \%$ of adolescents met the current physical activity (PA) guidelines of $60 \mathrm{~min}$ utes of aerobic PA a day [26]. Since $95 \%$ of youths spend 6-7 hours a day at school, this setting has been identified as an important institution for the promotion of PA and a great environment to give students opportunities to be active [4, 27]. Previous research has demonstrated that specific PA opportunities within the school day such as physical education (PE) and recess can make important contributions to children's overall PA [6]. While PA promotion is a specific role of the physical educator it is not expected that students meet the recommended 60 minutes of daily PA in PE class alone. PE teachers are recommended to strive for their students to be engaged in moderate-to-vigorous PA (MVPA) for at least $50 \%$ of the time spent in class [31]. Currently no PA guidelines exist for recess [21]. Although the school environment is an ideal setting to engage students in PA, overall research has indicated daily PA among youth is low and PA participation only declines with age [32]. Due to low daily PA participation, programs such as "Let's Move Active Schools" are campaigning to increase school PA time in the USA.

Engaging in PA is an integral part to obtaining and maintaining a healthy lifestyle [21]. Although most PA research focuses on benefits adults receive from PA, youth also receive specific benefits. Regular PA among youth leads to higher levels of aerobic fitness, stronger muscles, stronger bones and lower body fatness than inactive youth [30]. Additionally active youth have lower anxiety and depression [30]. The importance of engaging youth in PA is built on the premise that PA behavior tracks from childhood to adulthood [15]. This means that active youth will lead to active adults thereby reducing health problems associated with adult inactivity. Although the benefits of PA are well known, youth are becoming increasingly sedentary $[8,10,11$, $23,28]$. As student PA levels continue to drop, it is contributing to students performing lower in their fitness tests as they age [9].

Research studies have shown low associations with between aerobic fitness and PA among youth [13, 16, $17,19,22]$. It has been recommended that more studies 
should examine the relationships that exist between PA participation and aerobic fitness [3]. Furthermore this research should examine these relationships based on age and gender. To date, limited research findings have examined the relationship between elementary students' PA levels and aerobic fitness. It must be understood why students may or may not be meeting a certain aerobic fitness level and if it pertains to their involvement and participation in physical activity at school and outside of school. Therefore the purpose of this study was to examine if self-reported PA levels through the Physical Activity Questionnaire for Older Children (PAQ-C) and step-counts during PE as measured by pedometers of 4 th and 5 th grade students varied based on the students' field tests of cardiovascular fitness (i.e., number of laps completed).

\section{Material and methods}

\section{Participants and Setting}

All students $(N=94)$ in two 4th $(n=48 / \mathrm{F}-26$, M-22) and two 5th ( $n=46 / \mathrm{F}-22, \mathrm{M}-24)$ grades from a rural elementary school in the Midwestern USA were selected to participate using convenience sampling. Prior to the initiation of the study, approval was granted by the University Institutional Review Board (IRB), school administration, and the PE teacher. All students and legal guardians provided assent/consent to participate in the study. The average age of the students was 9.7 years. Demographics of the students at the school were reported as predominantly white (90\% Caucasian, 4\% Hispanic, 4\% Multi-Racial) middle-class to upper-class (5.8\% in 2010 and $12.8 \%$ in 2011 Low-Income) living in the community with many organized out of school opportunities for involvement in PA. Students received PE daily, with 4th graders attending a $25 \mathrm{~min}$ period and 5th graders attending a $30 \mathrm{~min}$ period. Class sizes varied from 20-25 students. One certified elementary PE specialist with five years of experience taught PE. The PE program was based upon teaching skills within a game setting rather than in isolation (e.g., Teaching Games for Understanding) [18].

\section{Instrumentation}

New Lifestyles SW-701 Digiwalker was utilized to assess the number of steps taken by students during five PE classes. The SW-701 was shown to have the smallest mean error $(-0.1)$ when compared with nine other pedometers and was accurate to \pm 17 steps of an average of 512 steps, or within $\pm 3 \%$ of the actual steps taken $95 \%$ of the time [24].

The PAQ-C is a self-administered, 7-day recall instrument. It was developed to assess general levels of
PA for students in grades 4 to 8 and approximately 8 to 14 years of age [12]. The PAQ-C provides a summary PA score derived from nine items, each scored on a 5-point scale.

The FitnessGram ${ }^{\circledR}$ health-related fitness assessment is a part of the district PE program requirements which assesses body composition, cardiovascular endurance, muscular strength/endurance, and flexibility [33]. To estimate students' aerobic capacity (cardiovascular endurance), the PACER test was performed.

\section{Procedures}

Data were collected over the course of two weeks. During the first week, students were instructed on how to properly attach, wear and use the pedometer. The students practiced the protocols of the study by picking up the pedometer, attaching, wearing, and returning their pedometer during each PE class that week. The students were given a chance to practice the PACER test routine and protocol, as a part of the curriculum requirements prior to the actual testing date. The $\mathrm{PE}$ teacher followed the administration procedures for the PACER as outlined in the FitnessGram ${ }^{\circledR}$ \& ActivityGram ${ }^{\circledR}$ test administration manual [33]. At the end of week one all students completed the FitnessGram ${ }^{\circledR}$ health-related fitness PACER test.

In week two, students step counts in five consecutive PE classes were recorded. The primary researcher observed each class to ensure that tampering of the pedometer was not taking place. If tampering was observed, the student was identified, as was the number of the pedometer he or she was using, and the data for that day was thrown out. Immediately following the conclusion of the lesson, a researcher recorded the steps from each pedometer on a data sheet, reset the pedometer to zero and returned it to the appropriate place in the box.

After PE class on the last day of data collection the students were led to another classroom to complete the researcher-led PAQ-C. The PAQ-C was then given out and all students were instructed to complete the demographic questionnaire on the first page that included information such as gender, age, and class. The researcher then verbally administered the questionnaire, and the students went through each question together as a group.

\section{Data Analysis}

Inclusion criterion for this study was data for 4 out of 5 days. Every student met that criterion. Descriptive statistics for the total sample and separate gender analyses were completed and are presented as means $\pm \mathrm{SD}$. Mean total/steps for each individual were computed as were mean scores computed for the PAQ-C. Independent samples $t$-tests were used to identify differences between boys and girls. 
Statistical analysis was completed using IBM SPSS (Statistical Package for the Social Sciences) Version 21 (Somers, NY). Assumptions for univariate and multivariate analysis (normality, linearity, and homoscedasticity) were checked prior to conducting the inferential statistics. All statistical analyses were conducted using a significance level of $p=0.05$. Three multiple regression analyses were conducted to predict the achievement on the aerobic capacity PACER test from the students mean average steps and their overall PAQ-C score. The independent variable of achievement on the PACER test is the raw score taken on the day the test was administered. The dependent variable of pedometer steps was specific to the average of the students' steps taken during their designated PE time, while the PAQ-C score was specific to the student's overall PA levels and their answers to the self-report questionnaire. The first regression included all students, while two additional multiple regressions were then computed to look for gender differences among the variables.

\section{Results}

Table 1 shows the means and standard deviation of the participants' average steps, PAQ-C and PACER results and Table 2 shows the regression results.

Table 1. Average Scores for Steps, PAQ-C, \& PACER (Mean \pm SD)

\begin{tabular}{lccc}
\hline & Steps & PAQ-C & PACER \\
\hline Males & $2203.4 \pm 361.1^{*}$ & $3.6 \pm 0.06$ & $36.6 \pm 14.8^{*}$ \\
Females & $1968.8 \pm 316.8$ & $3.4 \pm 0.71$ & $24.7 \pm 10.7$ \\
Total & $2083.6 \pm 357.3$ & $3.5 \pm 0.69$ & $30.5 \pm 14.1$ \\
\hline
\end{tabular}

Notes: Mean \pm Standard Deviation

*Indicates males are statistically different from females $(p<0.05)$
Results of the first regression analysis indicated that the students average steps and their PAQ-C score accounted for a significant amount of the PACER variability, $\mathrm{R}^{2}=0.36, \mathrm{p}=0.01$, indicating that students who scored higher on the PAQ-C and took more average steps achieved more laps during the PACER test. It was found that the average steps significantly predicted PACER laps $(\beta=0.48, \mathrm{p}<0.01)$, as did the PAQ-C $(\beta=0.28, p<0.001)$. Although both average steps and PAQ-C were significant, the relationship between the students PACER test and their average steps was stronger $(r=0.53, p<0.001)$ than their PACER test and their PAQ-C scores $(r=0.36, p<0.001)$.

When looking specifically at the males, the multiple regression indicated $\mathrm{R}^{2}=0.23 \mathrm{p}<0.005$. Both the average steps and the PAQ-C scores accounted for $23 \%$ of the variance in the PACER scores. It was found that average steps significantly predicted PACER laps ( $\beta=0.48, p<0.01)$ which is similar to the initial regression, while the PAQ-C and the PACER beta coefficients were not statistically significant $(\beta=0.14$, $\mathrm{p}=0.30$ ). This implies that when looking specifically at male PACER scores, their steps in class is a strong indicator of their PACER scores and that if one of the variables goes up the other variable should also increase.

The results of the multiple regression for females indicated $\mathrm{R}^{2}=0.41, \mathrm{p}<0.01$. The amount of variance accounted for in their PACER scores increased. These results show that when limited to just female scores, their PACER score were strongly associated to the combination of their average steps and their PAQ-C score. It was found that the average steps significantly predicted PACER laps $(\beta=0.38, \mathrm{p}<0.01)$, as did the PAQ-C $(\beta=0.46, \mathrm{p}<0.001)$. The females' coefficient for average steps was much lower than the males, indicating that the female scores brought down the overall total. The PAQ-C coefficient was much higher than the overall coefficient including both genders, but the

Table 2. Multiple regression model predicting PACER laps in boys and girls

\begin{tabular}{lccccc}
\hline & Unstandardized $\beta$ & $95 \%$ CI for $\beta$ & Standardized $\beta$ & $\mathrm{p}$ & Model $r^{2}$ \\
\hline Boys and Girls & & & & & 0.358 \\
Average Steps & 0.019 & $0.012,0.026$ & 0.484 & $<0.001$ & \\
PAQ-C & 5.78 & $2.30,9.27$ & 0.281 & 0.001 & \\
Boys & & & & & 0.231 \\
Average Steps & 0.019 & $0.008,0.030$ & 0.454 & 0.002 & \\
PAQ-C & 3.21 & $-2.97,9.40$ & 0.140 & 0.301 & \\
Girls & & & & & 0.410 \\
Average Steps & 0.013 & $0.005,0.021$ & 0.376 & 0.002 & \\
PAQ-C & 6.89 & $3.34,10.43$ & 0.456 & $<0.001$ & \\
\hline
\end{tabular}


males' coefficient was not statistically significant, so therefore it can be assumed that for females the association between their PACER scores and their PAQ-C score was stronger.

\section{Discussion}

This study examined whether self-reported PA levels and step-counts during PE of youth varied based on the students' number of laps completed during the FitnessGram PACER test. Elementary school students that took more steps in PE and reported higher PAQ-C scores tended to have higher PACER scores. When looking at gender specifically, the males' PACER scores were more strongly related to step counts in PE, while the females' scores were similarly related to both steps in $\mathrm{PE}$ and the PAQ-C scores. These results point to PE class time and the PE teacher's role in achieving maximum activity time as important factors in potentially improving their students' aerobic capacity (PACER scores). While it is important to increase class time step counts, it is still beneficial to encourage students to stay active outside of school. As per results of this study higher step counts in PE coupled with increased PA outside of PE was associated with higher PACER scores. Existing research studies with elementary school aged students $[5,17,22]$ have reported moderate relationships between PA and aerobic fitness. Le Masurier and Corbin [14] examined the relationship of step counts and aerobic fitness levels of middle school students and found those who had a higher amount of daily steps had higher fitness levels. Most recently, Kristensen et al. [13] examined the association of aerobic fitness and physical activity among European youth. Results confirmed a weak to moderate relationship of aerobic fitness and physical activity which is similar to the current and previous studies that were conducted among youth in the U.S.

Overall, this sample was highly active. The 4th and 5th grade students took 2.866.60 \pm 357.34 average steps in PE, which is substantially higher than the suggestion of 1.200 to 2.000 steps for an active 30 -minute PE class [30]. Tudor-Locke, et al. [29] found $6^{\text {th }}$ grade students took an average of 1,417 steps during a 30 minute PE period, while $7^{\text {th }}$ graders averaged 2.046 steps during a 50 minute PE class [7], with more time allotted in PE and lower step count averages than the current study.

The results of the current study reinforced the gender gap in daily PA participation. The females in our study took $1968.81 \pm 316.75$ steps, while the males took $2203.38 \pm 361.08$. A similar study by Scruggs et al. [25] quantified the steps of 3rd and 4th graders students $(\mathrm{n}=131)$ during a 30-minute PE class and they accumulated averages of 2.035 (males) and 1.858 (females) steps, which is more closely aligned with this sample. The students in the current study were still substantially more active than 4th and 5th grade children sampled across six schools, whose students accumulated only 1.662 and 1.581 steps for males and females [1].

The students averaged 3.52 on their PAQ-C, which shows that the students PA outside of PE was in the higher activity range (a perfect score of 5 being the highest). The males had an average score of $3.64 \pm 0.06$, while the females averaged $3.39 \pm 0.71$. These scores were also higher than a sample of 215 elementary aged students in the USA whose mean activity scores for females were $2.96 \pm 0.69$ and $3.44 \pm 0.68$ for males [2].

The students PACER laps were also considered high as the overall average was $30.52 \mathrm{SD} \pm 14.14$ with males completing $36.59 \pm 14.84$ and the females completing $24.71 \pm 10.68$. In a sample of 68 , males $(n=37)$ completed $31.24 \pm 12.93$, while females $(n=31)$ completed $23.61 \pm 10.75$ [34]. These scores may be higher than other studies due to the fact that the PAQ-C scores and step counts were higher than other research studies, which indicated that this sample was a highly active group. The male scores for PA were higher than the females thus it is not surprising that their PACER scores were also higher.

This sample was much different than other samples that have been previously examined due to the rural community, the opportunities for out of school extracurricular PA, and the above average PE class time per week. All these factors may have played a role in this sample achieving higher scores than other research samples in the USA. In this study, students attended $\mathrm{PE}$ every day of the week with 4th graders receiving a 25 minute lesson and 5 th grader receiving a 30 minute lesson. This adds up to 4th graders receiving $125 \mathrm{~min}-$ utes a week, just below the National Association for Sport and Physical Education [20] recommendation, with 5 th graders meeting the mark at 150 minutes per week. The minutes allotted for PE in the current study are still significantly higher than the national average of 85-95 minutes [27]. The higher than average minutes spent in PE coupled with the PE teachers' teaching style and the ample opportunities to be active provided by the rural community and teacher could be an explanation for the students' higher step counts, PAQ-C, and PACER laps.

By addressing all aspects related to PA, including the various opportunities outside of school available to their student body, fitness assessments, PAC-Q scores, and gender issues within their school, PE programs can advocate to promote PA within any setting. Future research needs to address the variances in elementary PE 
class time and the differences in these samples daily PA levels and fitness scores. Although the PAQ-C scores may not be as reliable, the average step counts were a valid measure of PA. It should be noted that future research should also compare self-reported PA outside of classes with an objective PA measurements such as pedometers or accelerometers. Additionally, other factors that are known to influence aerobic fitness such as body composition should be examined. Dencker et al. [5] examined body composition and vigorous-PA (VPA) as determinants of $\mathrm{VO}_{2}$ peak in youth between the ages of 8-11. Results indicated that body composition was a stronger predictor of $\mathrm{VO}_{2}$ peak than VPA. Looking forward it is essential that research continues to sample elementary students concerning PA inside and outside of the school setting so as to give all students all the necessary tools to achieve 60 minutes of PA, thus maintaining a lifestyle of health in childhood and adulthood.

\section{References}

1. Brusseau T.A., P.H. Kulinna, C. Tudor-Locke, M. Ferry, H. van der Mars, P.W. Darst (2011) The segmented physical activity patterns of elementary school children. J. Phys. Act. Health, 8: 279-286.

2. Crocker P.R.E., D.A. Bailey, R.A. Faulkner, K.C. Kowalski, R. McGrath (1997) Measuring general levels of physical activity: Preliminary evidence for the Physical Activity Questionnaire for Older Children. Med. Sci. Sports Exerc., 29: 1344-1349.

3. Cureton, K.J., G.L. Warren (1990) Criterion-referenced standards for youth health-related fitness tests: A tutorial. Res. Q. Exerc. Sport., 61: 7-19.

4. Dale D., C.B. Corbin, K.S. Dale (2000) Restricting opportunities to be active during the school day: Do children compensate by increasing physical activity levels after school? Res. Q. Exerc. Sport., 71: 240-248.

5. Dencker, M., O. Thorsson, M.K. Karlsson, C. Linden, S. Eiberg, P. Wollmer, L.B. Anderson (2007) Gender differences and determinants of aerobic fitness in children aged 8-11 years, 99: 19-26.

6. Fairclough S., G. Stratton (2006) A review of physical activity levels during elementary school physical education. J. Teach.Phys. Educ., 25: 239-257.

7. Flohr J.A., M.K. Todd, C. Tudor-Locke (2006) Pedometer-assessed physical activity in young adolescents. Res. Q. Exerc. Sport, 77: 309-315.

8. Gordon-Larsen P., M.C. Melson, B.M. Popkin (2004) Longitudinal physical activity and sedentary behavior trends: adolescence to adulthood. Am. J. Prev. Med., 27: 277-283.
9. Gidlow C., T. Cochrane, R. Davey, H. Smith (2008) In-school and out-of-school physical activity in primary and secondary school children. J. Sports Sci., 26(13): 1411-1419.

10. Kahn J.A., B. Huang, M.W. Gillman, A.E. Field, S.B. Austin, G.A. Colditz, A.L. Frazier (2008) Patterns and determinants of physical activity in U.S. adolescents. J. Adolesc. Health, 42: 369-377.

11. Kelder S.H., C.L. Perry, K.I. Klepp (1993) Community-wide youth exercise promotion: Long-term outcomes of the Minnesota Heart Health Program and the Class of 1989 Study. J. Scholastic Health, 63: 218-223.

12. Kowalski K.C., P.R.E. Crocker, R.M. Donen (2004) The Physical Activity Questionnaire for Older Children (PAQ-C) and Adolescents (PAQ-A) manual. Available at http://hkin.educ.ubc.ca/behavioural/PAQ\%20manual.pdf.

13. Kristensen P.L., N.C. Moeller, L. Korsholm, E. Kolle, N. Wedderkopp, K. Froberg, L.B. Andersen (2010) The asscociation between aerobic fitness and physical activity in children and adolescents: the European youth heart study. Eur. J. Appl. Physiol., 110: 267-275.

14. Le Masurier G.C., C.B. Corbin (2006) Step Counts Among Middles School Students Vary with Aerobic Fitness Level. Res. Q. Exerc. Sport., 77: 14-22.

15. Malina R.M. (2001) Adherence to physical activity from childhood to adulthood: a perspective from tracking studies. Quest., 53: 346-55.

16. Mathias, K., J.L. DePaepe, F. Konukman, S.C. Jefferies (2004) Investigation of the relationship between fitness and physical activity levels in middle school students. Res. Q. Exerc. Sport, 75: A-12.

17. Matsuzaka A., K. Matsuzaka, B. Wilk, O. Bar-Or (2003) Relationship between physical activity and aerobic fitness in children. Med. Sci. Sports Exerc., 35: S64.

18. Mitchell, S.A., J.L. Oslin, L.L. Griffin (2013) Teaching Sport Concepts and Skills ( $3^{\text {rd }}$ eds.). Human Kinetics, Champaign, IL.

19. Morrow, J.R., P.S. Freedson (2004) Relationship between habitual physical activity and aerobic fitness in adolescents. Pediatr. Exerc. Sci., 6: 315-329.

20. National Association for Sport and Physical Education and American Heart Association (2012) 2012 Shape of the Nation Report: Status of Physical Education in the USA. Reston, VA: American Alliance for Health, Physical Education, Recreation and Dance.

21. Ridgers N.D., G. Stratton, S.J. Fairclough (2005) Assessing physical activity during recess using accelerometry. Prev. Med., 41: 102-107.

22. Rowlands A.V., R.G. Eston, D.K. Ingledew (1999) Relationship between activity levels, aerobic fitness, and body fat in 8- to 10-year-old children. J. Appl. Physiol., 86: 1428-1435. 
23. Sallis J.F. (2000) Age-related decline in physical activity: a synthesis of human and animal studies. Med. Sci. Sports Exerc., 32: 1598-1600.

24. Schneider P.L., S.E. Crouter, O. Lukajic, D.R. Bassett (2003) Accuracy and reliability of 10 pedometers for measuring steps over a 400-m walk. Med. Sci. Sports Exerc., 35(10): 1779-1784.

25. Scruggs P.W., S.K. Beveridge, D.L. Watson, B.D. Clocksin (2005) Quantifying physical activity in firstthough 4th-grade physical education via pedometry. Res. Q. Exerc. Sport., 76: 166-175.

26. Synder T., S. Dillow (2011) Digest of education statistics 2010. Washington, DC: National Center for Education Statistics, Institute of Education Sciences, US Department of Education.

27. Snyder T.D., S.A. Dillow, C.M. Hoffman (2009) Digest 81. of education statistics 2008. Washington (DC): U.S. Department of Education, Institute of Education Sciences, National Center for Education Statistics. NCES publication number 2009-020.

28. Trost S.G., R.R. Pate, J.F. Sallis, P.S. Freedson, W.C. Taylor, M. Dowda, J. Sirard (2002) Age and gender differences in objectively measured physical activity in youth. Med. Sci. Sports Exerc., 34: 350-355.

29. Tudor-Locke C., S.M. Lee, C.F. Morgan, A. Beighle, R.P. Pangrazi (2006) Children's pedometer-determined physical activity during the segmented school day. Med. Sci. Sports Exerc., 38(10): 1732-1738.
30. US Department of Health and Human Services (2008) 2008 Physical Activity Guidelines for Americans. Washington (DC): U.S. Department of Health and $\mathrm{Hu}-$ man Services.

31. US Department of Health and Human Services (2010) Strategies to Improve the Quality of Physical Education. Washington, DC: US Department of Health and Human Services.

32. US Department of Health and Human Services (2012) Physical Activity Guidelines for Americans Midcourse Report: Strategies to Increase Physical Activity Among Youth. Washington, DC: U.S. Department of Health and Human Services.

33. Welk G.J., M.D. Meredith (2008) Fitnessgram/ Activitygram Reference Guide. Dallas TX: The Cooper Institute; 2008, p. 27-51, 96-120, 169-178.

34. Welk G.J., M. Ihmels, J.J. McClain, J. Schaben (2006) The Reliability and convergent validity of field tests of body composition in young adolescents. J. Phys. Activ. Health, 3(Suppl 2): S67-S77.

\section{Received 05.08.2013 \\ Accepted 24.11.2013}

(C) University of Physical Education, Warsaw, Poland 